\title{
Optimization of the Revised Tokuhashi Scoring System: New Prognostic Criteria for Metastatic Spinal Tumor in Surgical Cases
}

\author{
Masahiro Iinuma, Tsutomu Akazawa, Yoshiaki Torii, Jun Ueno, Tasuku Umehara, Kota Asano, Shingo Kuroya, \\ Atsuhiro Yoshida, Yoshimichi Onose, Ken Tomochika and Hisateru Niki
}

Department of Orthopaedic Surgery, St. Marianna University School of Medicine, Kawasaki, Japan

\begin{abstract}
:
Introduction: The revised Tokuhashi scoring system has been used to predict survival in patients with metastatic spinal tumors. Because of the rapid progress of cancer therapy, the original criteria of the revised Tokuhashi scoring system became in recent years unsuitable. The study aim was to evaluate the validity of the revised Tokuhashi scoring system in patients who underwent spinal surgery and to establish new prognostic criteria.

Methods: The study enrolled 85 patients with metastatic spinal tumors who underwent spinal surgery. The patients' survival outcomes in October 2019 were as follows: 57 patients died; 10 were alive; and 18 had unknown prognoses. The study evaluated the validity of the Tokuhashi scoring system, and established and validated the new prognostic criteria.

Results: The accuracies of the Tokuhashi scoring system were $66.7 \%$ in the short-term group, $60 \%$ in the midterm group, and $100 \%$ in the long-term group. Among the patients who died, the survival period and total score were significantly correlated. Total score cutoff point was six points in the patients whose predicted survival was $<6$ months. Total score cutoff point was eight points in the patients whose predicted survival was $\geq 1$ year.

Conclusions: As the prognosis of patients has improved in recent years, the original criteria of the revised Tokuhashi scoring system have been questioned as to their suitability to current treatments. Especially, the survival period among the patients with total scores of 7 and 8 points was not accurate. According to this study, the new prognostic criteria of the revised Tokuhashi scoring system were set to 0 to 6 points for the short-term group, 7 points for the midterm group, and 8 to 15 points for the long-term group.
\end{abstract}

Keywords:

Revised Tokuhashi Score, metastatic spinal tumors, life prognosis

Spine Surg Relat Res 2021; 5(2): 81-85 dx.doi.org/10.22603/ssrr.2020-0102

\section{Introduction}

Spinal surgery for metastatic spinal tumors has the possibility to improve patient quality of life and performance status. Prediction of prognosis strongly influences surgical indications and procedures. Tokuhashi et al. in their study reported the revised Tokuhashi scoring system in 2005 and the system has been widely used to predict survival in patients with metastatic spinal tumors ${ }^{1)}$. Several papers have reported the usefulness of the revised Tokuhashi scoring system $^{2-4)}$. Based on the original criteria of predicted prognosis, total score of the revised Tokuhashi scoring system was 0 to 8 points as the short-term group (predicted survival: $<6$ months), 9 to 11 points as the midterm group (predicted sur- vival: $\geq 6$ months), and 12 to 15 points as the long-term group (predicted survival: $\geq 1$ year) ${ }^{1}$. As the prognosis of patients has improved in recent years, the original criteria of the revised Tokuhashi scoring system have been questioned as to their suitability to current treatments ${ }^{5-7)}$. The aims of this study were to evaluate the validity of the revised Tokuhashi scoring systems in patients who underwent spinal surgery for metastatic spinal tumors and to establish new prognostic criteria.

\section{Materials and Methods}

The institutional review board of our institution approved this retrospective study. The study enrolled 85 patients (48

Corresponding author: Masahiro Iinuma, jspfy033@yahoo.co.jp

Received: May 25, 2020, Accepted: July 11, 2020, Advance Publication: August 31, 2020

Copyright (C) 2021 The Japanese Society for Spine Surgery and Related Research 
men and 37 women; mean age of 62.4 years at the time of surgery; range of 26-85 years) from May 2009 to July 2018 with metastatic spinal tumors who underwent spinal surgery. The patients' outcomes in October 2019 were as follows: 57 patients died; 10 were alive; and 18 had unknown prognoses. The primary lesions were breast cancer in 22 patients, prostate cancer in 14 patients, lung cancer in 14 patients, hepatocellular carcinoma in seven patients, renal cell carcinoma in seven patients, gastric cancer in four patients, colon cancer in four patients, pancreatic cancer in three patients, thyroid cancer in two patients, bile duct cancer in two patients, ureteral cancer in two patients, malignant melanoma in one patient, duodenal cancer in one patient, bladder cancer in one patient, and primary unknown cancer in one patient. The surgical levels were the cervical spine in 21 patients, thoracic spine in 47 patients, and lumbosacral spine in 17 patients. The primary lesions in the deceased patients were breast cancer in 15 patients, lung cancer in 10 patients, prostate cancer in six patients, renal cell cancer in six patients, hepatocellular carcinoma in four patients, gastric cancer in three patients, colon cancer in three patients, pancreatic cancer in two patients, bile duct cancer in two patients, thyroid cancer in one patient, ureteral cancer in one patient, malignant melanoma in one patient, duodenal cancer in one patient, bladder cancer in one patient, and primary unknown cancer in one patient.

We performed surgeries in patients who had neurological deficit or severe pain due to spinal metastasis and the patients and their families understood the risk of operation. The surgical procedures were posterior decompression in six patients, posterior fixation in 71 (including 69 patients with decompression), anterior fixation in one, anteroposterior fixation in five (including two patients with decompression), balloon kyphoplasty in one, and biopsy in one. In major complications, there were five cases with surgical site infection, three cases with recurrence of tumor requiring additional surgery, two cases with postoperative hematoma, and one case with rod fracture.

Survival period was defined as duration from the day of operation to the date of death or the latest follow up.

The study evaluated the validity of the revised Tokuhashi scoring system, establishing new prognostic criteria, and evaluating their validity.

\section{Validity of the revised Tokuhashi scoring system}

Based on the original criteria of the revised Tokuhashi scoring system, all patients were classified into the shortterm group (total score 0-8 points, predicted prognosis: $<6$ months), midterm group ( $9-11$ points, predicted prognosis: $\geq 6$ months), and long-term group (12-15 points, predicted prognosis: $\geq 1$ year). The prognoses between the groups were examined by performing the Kaplan-Meier curve analysis and the log-rank test. Subsequently, the correlation between total score and true prognosis was evaluated by the Spearman's rank correlation coefficient in 57 deceased patients.

\section{New prognostic criteria in deceased patients}

In the 57 deceased patients, receiver operating characteristic (ROC) curves were used to examine the grouping that gave the highest concordance rate for the short-term group defined as $<6$ months, the midterm group as $\geq 6$ months, and the long-term group as $\geq 1$ year. New prognostic criteria were created.

\section{Validity of the new prognostic criteria in all patients}

The new prognostic criteria were applied to all 85 patients. We examined whether the median days of survival in the short-term, midterm, and long-term groups were stratified using the Kaplan-Meier curve analysis and the log-rank test.

All statistical analyses were performed using the EZR software (Saitama Medical Center, Jichi Medical University, Saitama, Japan) $)^{8}$. The Kaplan-Meier curves, log-rank tests, ROC curves, and Spearman's rank correlation coefficients were used for statistical analyses, and the level of significance was set at $5 \%$.

\section{Results}

\section{Validity of the revised Tokuhashi scoring system}

According to the original criteria of the revised Tokuhashi scoring system, patients were divided into a short-term group (total score 0-8 points; 57 patients), midterm group (total score 9-11 points; 22 patients), and long-term group (total score $12-15$ points; 6 patients). The median survival periods were 5.9 months in the short-term group, 30.1 months in the midterm group, and 38.8 months in the longterm group. The accuracies of the original criteria of the revised Tokuhashi scoring system were $66.7 \%$ in the shortterm group, $60 \%$ in the midterm group, and $100 \%$ in the long-term group. For these three groups, the log-rank test using the Kaplan-Meier curves showed significant differences between the groups $(P=0.03)$ (Fig. 1). Among the patients who died, total score and the survival period were significantly correlated $(r=0.473, P<0.01)$ (Fig. 2).

\section{New prognostic criteria in deceased patients}

Total score cutoff point, based on whether or not the predicted prognosis was $<6$ months when using ROC analysis, was 6 points (sensitivity: $80.0 \%$; specificity: $62.5 \%$; area under the curve [AUC]: 0.741) (Fig. 3). Total score cutoff point, based on whether or not the predicted prognosis was $\geq 1$ year when using ROC analysis, was 8 points (sensitivity: 60.5\%; specificity: 89.5\%; AUC: 0.789) (Fig. 4). From these results, the new prognostic criteria were set as follows: total score 0 to 6 points=short-term group (predicted prognosis: < 6 months); total score 7 points=midterm group (predicted prognosis: $\geq 6$ months); and total score 8 to 15 points=longterm group (predicted prognosis: $\geq 1$ year) (Table 1 ). 


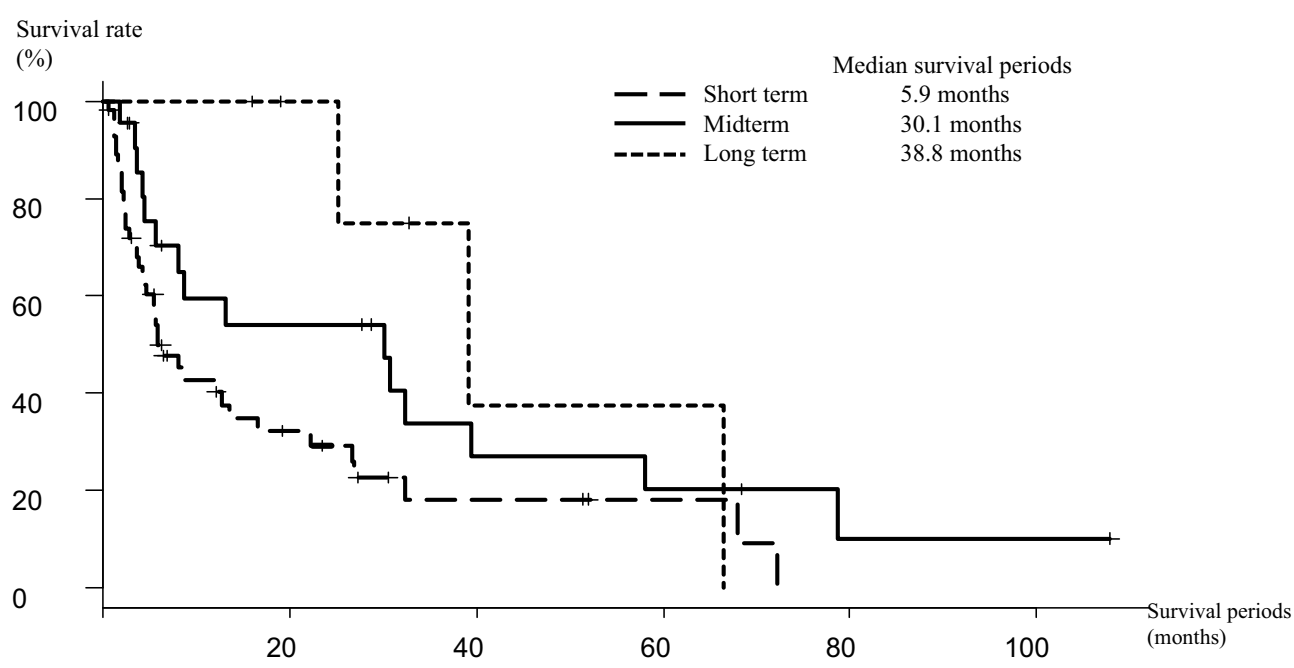

Figure 1. The Kaplan-Meier curves of the revised Tokuhashi scoring system in all patients.

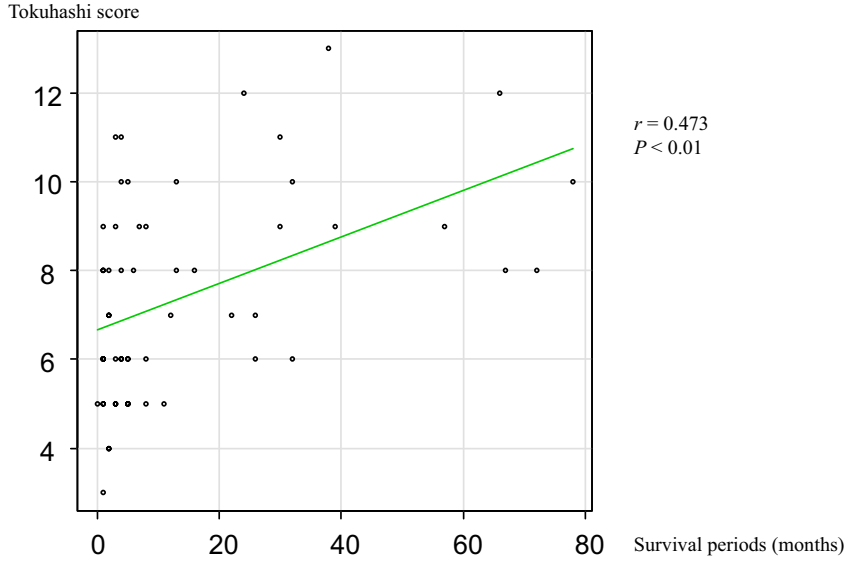

Figure 2. Correlation between total score and the survival period in deceased patients.

\section{Validity of the new prognostic criteria in all patients}

According to the new prognostic criteria, the median survival periods were 4.6 months in the new short-term group (31 patients), 12.8 months in the new midterm group (eight patients), and 32.3 months in the new long-term group (46 patients). The survival periods of these three groups were significantly different in the Kaplan-Meier curve analysis and log-rank test $(P<0.01)$ (Fig. 5).

\section{Discussion}

Published papers have shown the usefulness of the revised Tokuhashi scoring system ${ }^{2-4}$. Since cancer therapy has progressed with new chemotherapy and immune checkpoint inhibitors, the five-year survival rate of cancer patients has increased $^{6,7)}$. In recent years, Kitamura et al. reported that the lifetime prognoses of recent cohorts who underwent surgical treatment for metastatic spinal tumors are twice that of a previous cohort ${ }^{5}$. Since the original criteria of the revised Tokuhashi scoring system have been questioned as to their

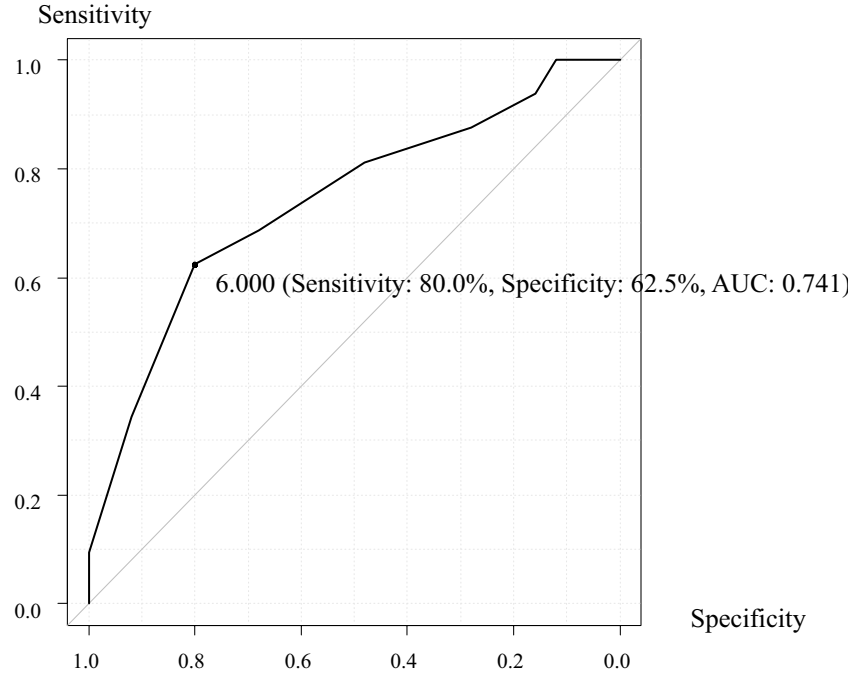

Figure 3. Total score cutoff point, based on whether or not the predicted prognosis was $<6$ months according to the ROC analysis, was 6 points.

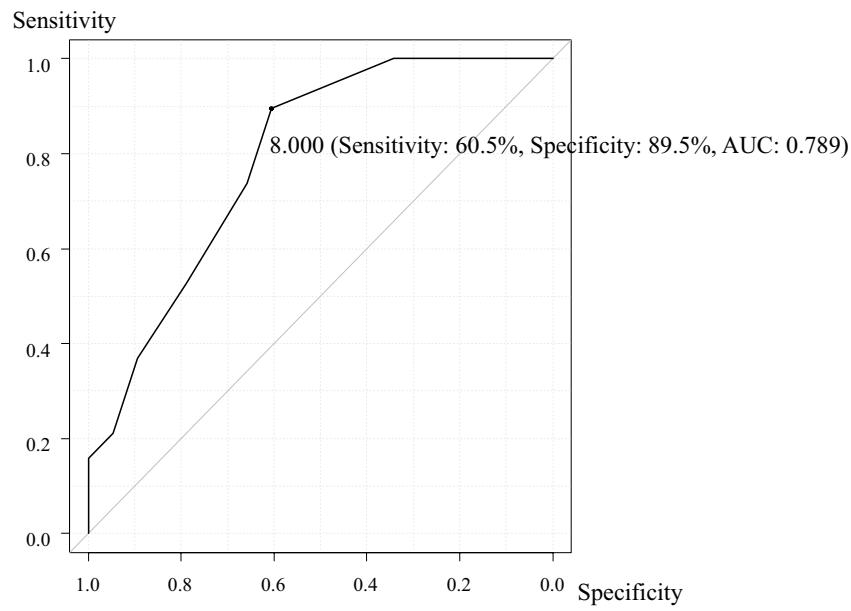

Figure 4. Total score cutoff point, based on whether or not the predicted prognosis was $\geq 1$ year according to ROC analysis, was 8 points. 
suitability, several papers have evaluated the validity of revised Tokuhashi scoring system to establish a new scoring system $^{9-11)}$. Westermann et al. evaluated the validity of the revised Tokuhashi scoring systems and set the new prognostic criteria which satisfied patients' prognoses in 1,3, and 12 months with ROC analysis ${ }^{9}$. Recent studies have suggested a new revision of the revised Tokuhashi scoring system. With Morgen et al. reporting another revision of the revised Tokuhashi scoring system which adjusted primary diagno$\operatorname{ses}^{10}$. In this study, total score of the revised Tokuhashi scoring system and the survival period were significantly correlated, with Hessler et al. concluding that the revised Tokuhashi scoring system is underestimating the life expectancy of patients with lung cancer ${ }^{11}$. We suggest that the prognostic prediction using the revised Tokuhashi scoring system has been useful in recent patients and been able to create new criteria. Total scores of 7 and 8 points were prolonged, and were regarded as short-term groups according to the original criteria. Finally, the new prognostic criteria were set as follows: short-term group, total score 0 to 6 points; midterm group, total score 7 points; and long-term group, total score 8 to 15 points.

This study has several limitations. 1) We only examined surgical patients. Because the prognosis was extremely short for some patients, such as those with poor performance status, patients with conservative treatment were not included. In contrast, the patients without symptoms were also

Table 1. New Prognostic Criteria of the Revised Tokuhashi Scoring System.

\begin{tabular}{lc}
\hline \multicolumn{1}{c}{ Total score } & Predicted prognosis \\
\hline 0 to 6 points & $<6$ months \\
7 points & $\geq 6$ months \\
8 to 15 points & $\geq 1$ year \\
\hline
\end{tabular}

not included. From these factors it can be seen that there is a possibility that the prognosis of this study does not reflect the true prognosis. 2) Because of the small number of patients, we did not examine each type of cancer and the rate of primary tumor. Further studies are required to investigate each type of cancer. 3) We had some major complications, but we did not investigate whether these major complications influenced patient's prognosis. 4) We did not investigate about preoperative comorbidities. It may influence patients' survival period. 5) Total points of the revised Tokuhashi score and patients' survival were significantly correlated. This result suggested that each point of the primary site still had validity. However, since survival periods of some primary cancers such as lung cancer are getting to be longer in recent years, it may change the point distribution. 6) This study was a retrospective study, and the protocol of postoperative therapy was not the same. It may influence the prognosis of the patient.

\section{Conclusion}

The total score of the revised Tokuhashi score and the survival period were significantly correlated. With improvement in cancer therapy in the recent years, the original criteria of the revised Tokuhashi scoring system were not accurate, particularly among the patients with total scores of 7 and 8 points. According to this study, new prognostic criteria of the revised Tokuhashi scoring system were set to 0 to 6 points for the short-term group (predicted prognosis: $<6$ months), 7 points for the midterm group (predicted prognosis: $\geq 6$ months), and 8 to 15 points for the long-term group (predicted prognosis: $\geq 1$ year).

Conflicts of Interest: The authors declare that there are no relevant conflicts of interest.

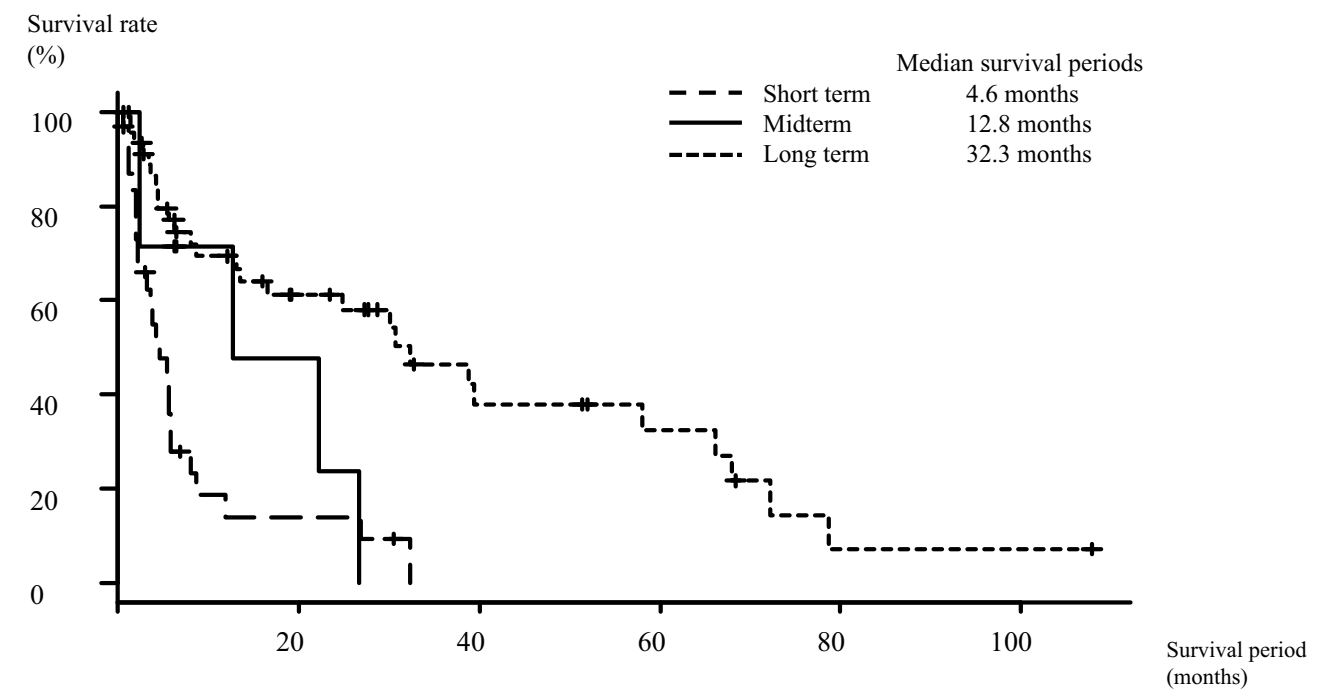

Figure 5. The Kaplan-Meier curves of new prognostic criteria of the revised Tokuhashi scoring system in all patients. 
Ethical Approval: No. 4182; Institutional review board of St. Marianna University School of Medicine.

\section{References}

1. Tokuhashi $\mathrm{Y}$, Matsuzaki $\mathrm{H}$, Oda $\mathrm{H}$, et al. A revised scoring system for preoperative evaluation of metastatic spine tumor prognosis. Spine. 2005;30(19):2186-91.

2. Yamashita T, Siemionow KB, Mroz TE, et al. A prospective analysis of prognostic factors in patients with spinal metastases. Spine. 2011;36(11):910-7.

3. Moon KY, Chung CK, Jahng TA, et al. Postoperative survival and ambulatory outcome in metastatic spinal tumors: prognostic factor analysis. J Korean Neurosurg Soc. 2011;50(3):216-23.

4. Chen H, Xiao J, Yang X, et al. Preoperative scoring systems and prognostic factors for patients with spinal metastases from hepatocellular carcinoma. Spine. 2010;35(23):1339-46.

5. Kitamura D, Hozumi T, Oka H, et al. Analysis of prognostic scoring systems in metastatic spinal tumors: Tokuhashi score, Katagiri score and Tomita score. J Spine Res. 2017;8(1):34-8. Japanese.

6. National Cancer Center Japan. Monitoring of Cancer Incidence in Japan 2016. Available from: https://ganjoho.jp/reg_stat/statistics/br ochure/ncr_incidence.html
7. Matsuda T, Ajiki W, Marugame T, et al. Population-based survival of cancer patients diagnosed between 1993 and 1999 in Japan: a chronological and international comparative study. Jpn J Clin Oncol. 2011;41(1):40-51. Japanese.

8. Kanda Y. Investigation of the freely available easy-to-use software 'EZR' for medical statistics. Bone Marrow Transpl. 2013;48(3): 452-8.

9. Westermann L, Olivier AC, Samel C, et al. Analysis of seven prognostic scores in patients with surgically treated epidural metastatic spine disease. Acta Neurochir (Wien). 2020;162(1):109-19.

10. Morgen SS, Fruergaard S, Gehrchen M, et al. A revision of the Tokuhashi revised score improves the prognostic ability in patients with metastatic spinal cord compression. J Cancer Res Clin Oncol. 2018;144(1):33-8.

11. Hessler C, Vettorazzi E, Madert J, et al. Actual and predicted survival time of patients with spinal metastases of lung cancer. Spine. 2011;36(12):983-9.

Spine Surgery and Related Research is an Open Access journal distributed under the Creative Commons Attribution-NonCommercial-NoDerivatives 4.0 International License. To view the details of this license, please visit (https://creativeco mmons.org/licenses/by-nc-nd/4.0/). 